\title{
Low-Cost Metamaterial Loaded Microstrip Antenna for Defense Applications
}

\author{
Krushi Modi*, Parth Kachhadiya, Jagdish M. Rathod, Parul H. Panchal, Kush Parikh \\ Electronics Engineering Department, BVM Engineering, Gujarat, India \\ Email: ^krushimodi97@gmail.com
}

How to cite this paper: Modi, K., Kachhadiya, P., Rathod, J.M., Panchal, P.H. and Parikh, K. (2021) Low-Cost Metamaterial Loaded Microstrip Antenna for Defense Applications. Open Journal of Antennas and Propagation, 9, 1-10. https://doi.org/10.4236/ojapr.2021.91001

Received: January 15, 2021

Accepted: March 28, 2021

Published: March 31, 2021

Copyright ( 2021 by author(s) and Scientific Research Publishing Inc. This work is licensed under the Creative Commons Attribution International License (CC BY 4.0).

http://creativecommons.org/licenses/by/4.0/

\begin{abstract}
We have designed a Metamaterial unit-cell for $9 \mathrm{GHz}$ frequency. Periodic structure was used at $4.25 \times 4.25 \mathrm{~mm}$ with a thickness of $0.35 \mathrm{~mm}$ and giving us the $99.99 \%$ of absorbance at $9 \mathrm{GHz}$ in simulated results. We have implemented a rectangular microstrip antenna and loaded it with Metamaterial unit-cells which provided improved results. There were results available for reflection coefficient (s11 parameter) at $9 \mathrm{GHz}$ and also helping for the reduction of the Radar Cross Section of an antenna, which reduced more than $20 \mathrm{~dB}$ and not affected its directivity and gain.
\end{abstract}

\section{Keywords}

Microstrip Antenna (MSA), Metamaterial Absorber (MMA),

Single Band MMA, Radar Cross Section (RCS), X-Band

\section{Introduction}

Nowadays, as researchers are trying to achieve electromagnetically different characteristics than the conventional materials and so the material entered and it's a Metamaterials. It has been proved to be very effective here. Metamaterial is basically periodic structures which show change in characteristics than normal material while resonating at certain frequencies [1]. Which provides us a whole new area of research to understand the characteristics of materials. So, with help of these new properties of material we can achieve number of goals like, to reduce the Radar cross section for stealth technologies and to make clocking devices and many more advance technologies. The reduced RCS of an antenna will help to achieve secrecy of an antenna to be less detectable in enemy radar section.

MMA's properties offer's us magnificent features, that includes near-unity 
absorbance, extremely-thin thickness with wide-range angles of incidence and polarization insensitivity, felicitous for antennas designs, for the reduction of radar cross section (RCS) in stealth technology by enhancing absorption to improve the performance and also useful in wireless communication technologies [2].

Metamaterials are widely used in with the antenna designs are having the negative refractive index. Negative refractive index is one of the most significant characteristics of the metamaterial. The phase of signal advances as its moves away from the source and fleeting waves increase as they get further away from the source, which shows us the difference between conventional material and metamaterial properties. In normal or conventional material $\mathrm{E}$-field and $\mathrm{H}$-fields creates a right-handed triplet with the direction of phase propagation. In negative refracted material has negative $\mu$ and negative $\mathcal{E}$, they create a left hand triplites and also known as left-handed materials [3], which are not been found in nature but can be able to produce artificially [4]. Wide research is going on in this field, researchers and engineers are getting more capable to design or create these types of material as never before.

\section{Microstrip Antenna without Metamaterial}

We are using very simple MSA configuration consisting of radiating patch on the top of dielectric substrate and ground plane at bottom. Also, it is more suitable for these types of metamaterial loaded antennas because of intelligible structure, linearly polarized, conformable to planar and non-planar structures, less expanses, can easily implementable and reconcilable with the PCB technology [5]. All the parameter of a rectangular microstrip patch antenna is calculated with the help of standard equations, based on studies of Balanis. We Obtained parameters as an optimized design of patch's length and width of $6.96 \mathrm{~mm}$ and $7.23 \mathrm{~mm}$, respectively. Thickness of patch is $0.035 \mathrm{~mm}$. A dielectric substrate is having a thickness of $h_{1}=1.35 \mathrm{~mm}$ in centre and in other area thickness $h_{2}=$ $0.35 \mathrm{~mm}$ for the better performance while loaded it with metamaterial and used FR-4 epoxy $\left(\varepsilon_{\gamma}=4.4, \tan \delta=0.02\right)$ as a dielectric material. The top view and a side view have been given in Figure 1.

All parameters calculated at $9 \mathrm{GHz}$ are given in Table 1 .

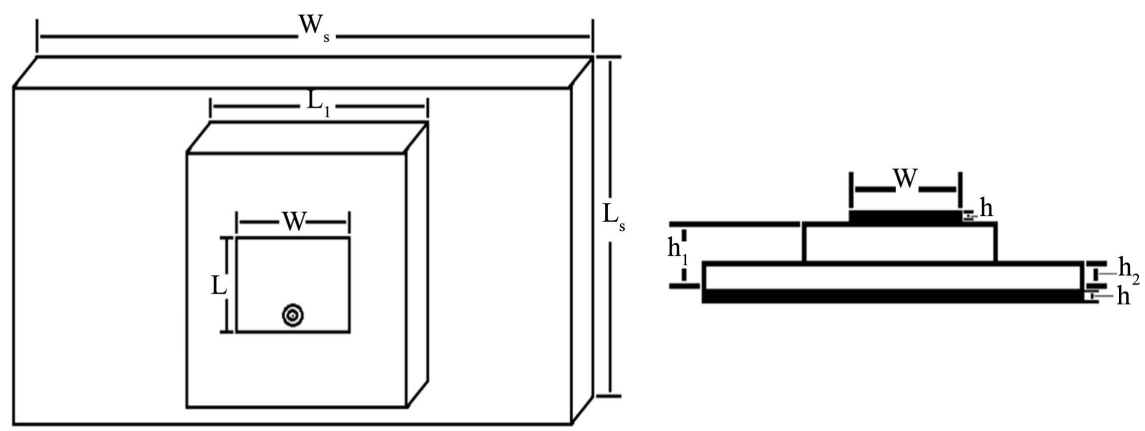

Figure 1. Top view and side view of MSA. 
Table 1. Description of all dimensions.

\begin{tabular}{ccc}
\hline Description & Dimensions $(\mathrm{mm})$ & Parameter \\
\hline$L_{s} / w_{s}$ & $29.75 / 67.5$ & Periodicity of the structure \\
$L_{1}$ & 12.5 & Central substrate length \\
$h_{1}$ & 1.35 & Height of central substrate \\
$h_{2}$ & 0.35 & Height of substrate \\
$L$ & 6.96 & Length of patch \\
$W$ & 7.23 & Width of patch \\
$H$ & 0.035 & Height of patch \\
\hline
\end{tabular}

At the bottom copper ground is given with the thickness of $0.035 \mathrm{~mm}$. Coaxial cable is used to feed the antenna at a point where it is giving better matching impedance around $50 \Omega$. This structure has been made with commercial software HFSS V 19. Simulated results of simple microstrip antenna the scattering coefficient $\left(s_{11}\right)$ without loading metamaterial is achieved to be $-36.33 \mathrm{~dB}$ at 9 GHz. Metamaterial are basically artificially designed periodic structures which shows different electro-magnetic properties than the conventional material. Metamaterials have a negative refractive index which is not found in natural material, which is first postulated by Veselago in 1968 [6] demonstrated in Figure 2.

These designed periodic structures are resonating at certain frequencies and able to absorb the electromagnetic radiation in the form of light. The theoretical background based on the negative refractive index of values of $\mathcal{\varepsilon}$ (permittivity) and $\mu$ (permeability).

Which is given as:

$$
\eta=-\sqrt{\mu_{r} \varepsilon_{r}} .
$$

And Pendry introduced the idea of artificial magnetism [7], that opened up the possibility of creating negative index material [8] and after that number of researches came out to support this concept and new era of metamaterial begun. In 2008, Landy [9] et al. proposed the first-ever perfect metamaterial absorber (MMA). By designing electric resonator on the top plane and cut wire on the bottom plane of the substrate, the absorber was able to get absorption closely 99\% at a single band frequency. After that, the research on absorbers has obtained prodigious amount of development, such as single-band [10], dual-band [11] [12], triple band [13], multi-band [14] [15], broadband [16] and tunable absorption [17]. Typically, metamaterial components are fashioned from the highly conductive materials like, gold, silver, copper and formed into the periodic structure to get desired characterization of EM properties.

Different types of periodic structures are designed at different frequencies to achieve good absorption and the rate of absorption can be achieved up to $100 \%$ with necessary height of substrate and structure of material designed [18]. And can be combined with the rectangular patch antenna to improve the performances. MMA loaded antenna can give more improved gain and directivity or keep them as it is [19]. Metamaterial absorber are widely used to reduce the radar cross section (RCS). 


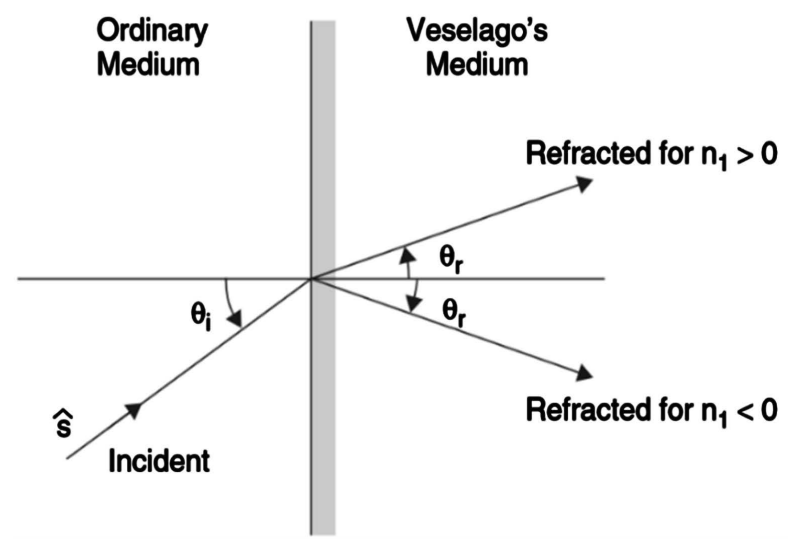

Figure 2. Presentation of Negative refractive index.

\section{Proposed Antenna Design with Metamaterial}

In proposed unit cell design, we have taken a unit cell of $4.25 \times 4.25 \mathrm{~mm}$, which is very compact in size. FR-4 epoxy is used as dielectric having $\varepsilon_{\gamma}=4.4, \tan \delta=$ 0.02 and the height of $0.35 \mathrm{~mm}$ with the copper as a metallic part. The gap between two-unit cell is $0.15 \mathrm{~mm}$. The unit cell is shown in Figure 3. The unit cell structure is consisting of rectangular rings and triangle, which looks like a charismas tree. The back surface of the given unit cell structure is made of metal, to ensure the zero transmission on the other side of structure [18] [19]. Back radiation or transmission is terminated the transmission coefficient $s_{21}(\omega)=0$. And the reflection from the structure needs to be decrease [20].

The proposed structure is simulated in HFSS V 19 software as it provides nice performance for similar structure [21]. The Floquet port and master-slave boundary conditions are utilized to simulate this finite array structure of Metamaterial. As shown in Figure 3. The absorption is taking place at a $9 \mathrm{GHz}$ frequency and absorption obtained through simulation is $99.99 \%$ as shown in the figure. Here, we have taken arrays of 7 by 7 for analysis [22].

The absorption of the unit cell is given by the equation:

$$
A(\omega)=1-R(\omega)^{2}-T(\omega)^{2}
$$

where, $A(\omega)=$ Absorption.

$R(\omega)=$ Reflected power.

$T(\omega)=$ Transmitted power.

Now MSA with loaded MMA have been explained. Loading the microstrip antenna with metamaterial cells which is shown in Figure 4. The Metamaterial cells are loaded on the substrate having a height of $0.35 \mathrm{~mm}$ [23]. The length and width of the lower substrate is $29.75 \mathrm{~mm}$ for 7 by 7 , shown in Figure 4 .

The absorption plot after loading the MSA with MMA is as given in Figure 5.

We can see that after loading the MSA with MMA it doesn't affect the gain and directivity as they are very similar to MSA results, as scattering parameter, Gain and Directivity comparison is given in Figure 6 with and without MMA loaded MSA [24] [25]. 


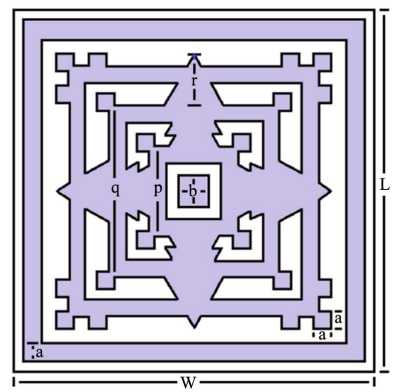

(a)

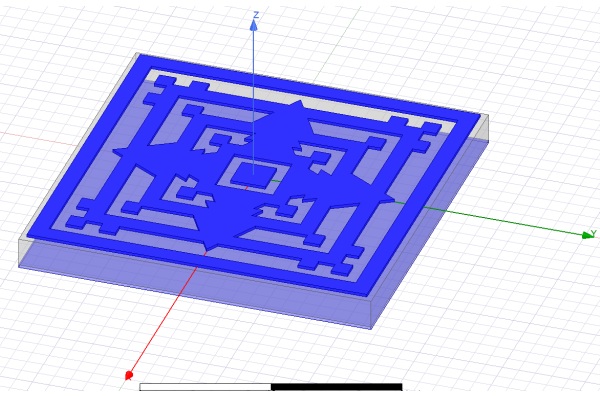

(b)

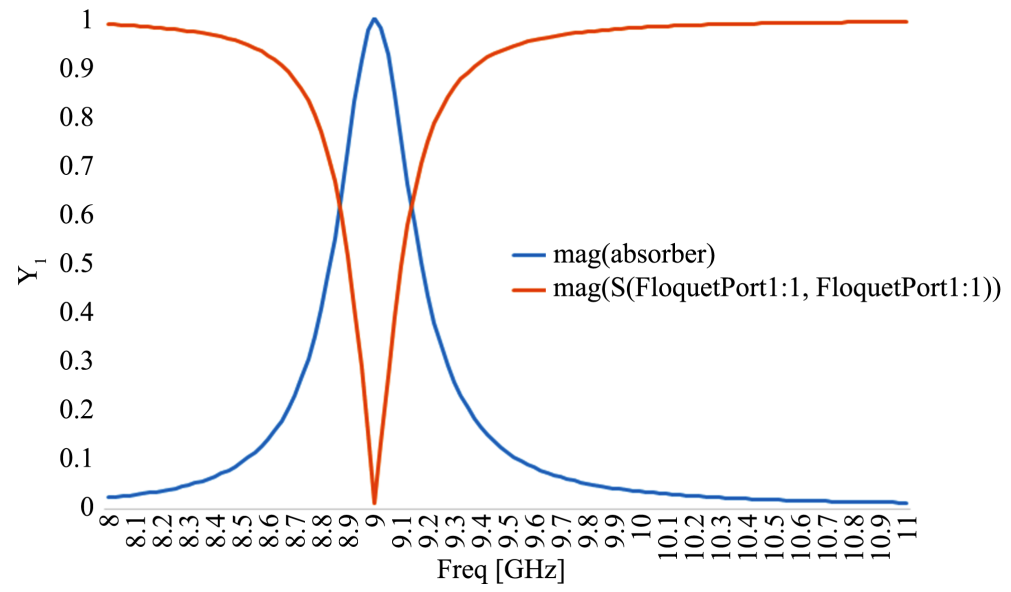

(c)

Figure 3. (a) Unit cell top view; (b) Trimetric view of unit cell; (c) Absorption plot of MMA.
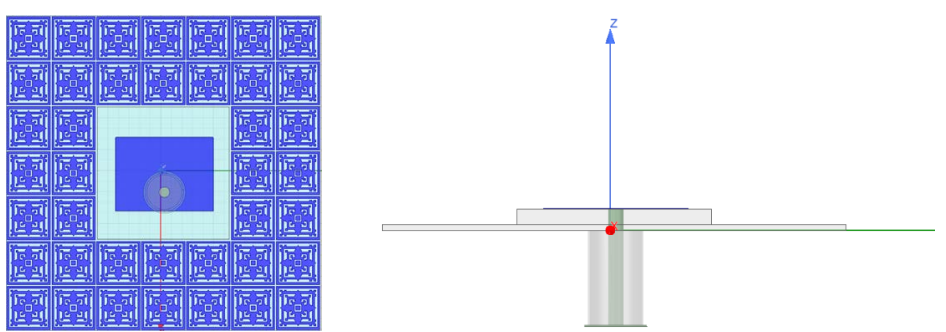

Figure 4. MSA loaded with MMA and side view of MMA loaded MSA.

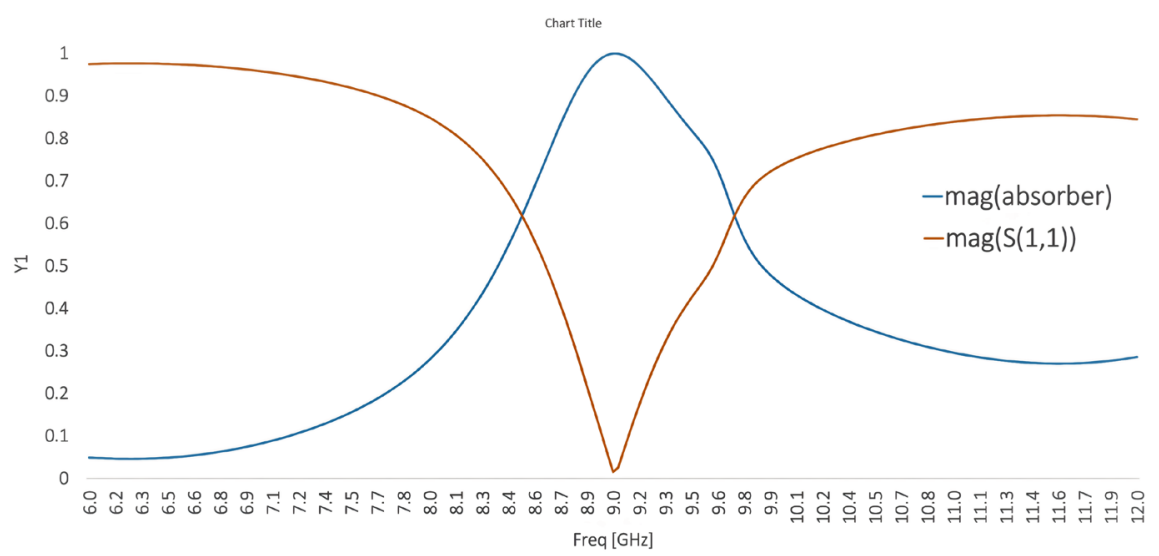

Figure 5. Absorption plot of MSA loaded with MMA. 


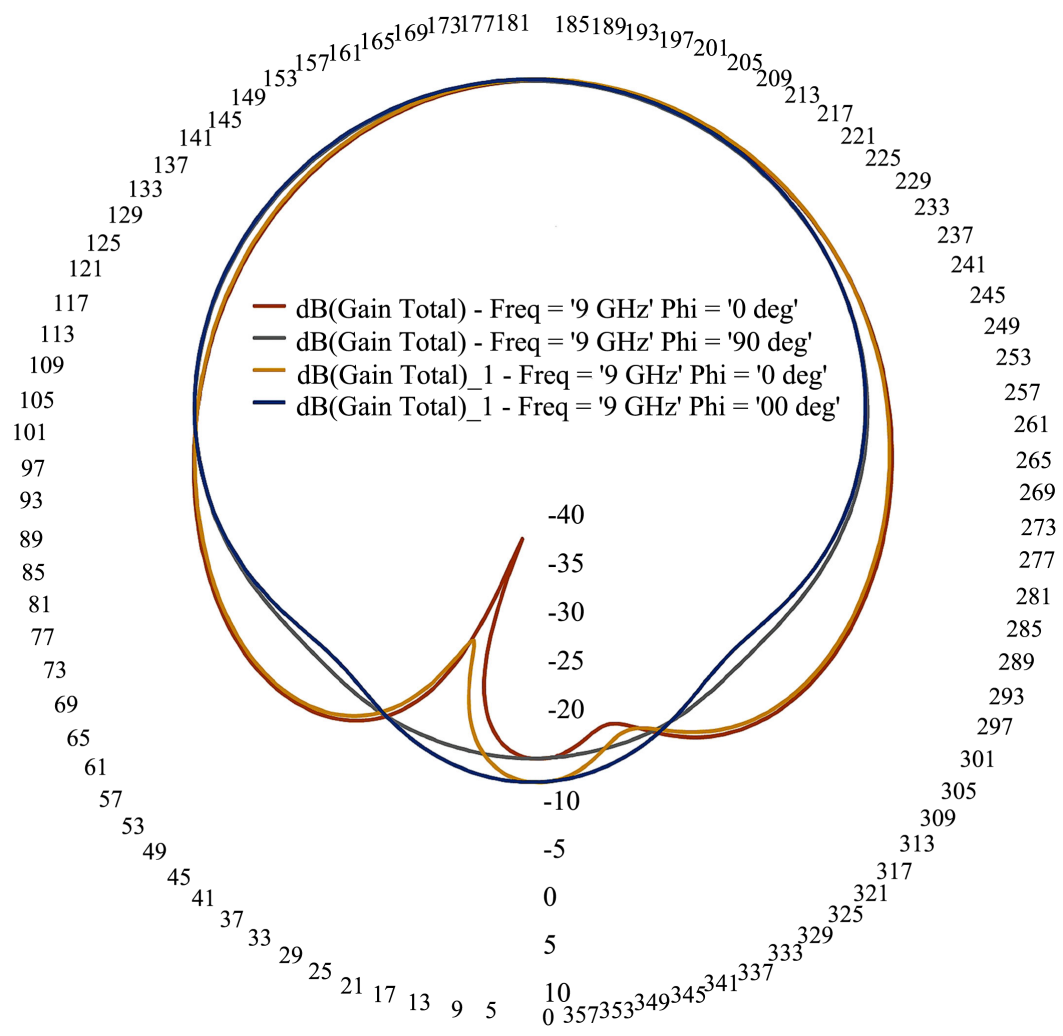

(a)

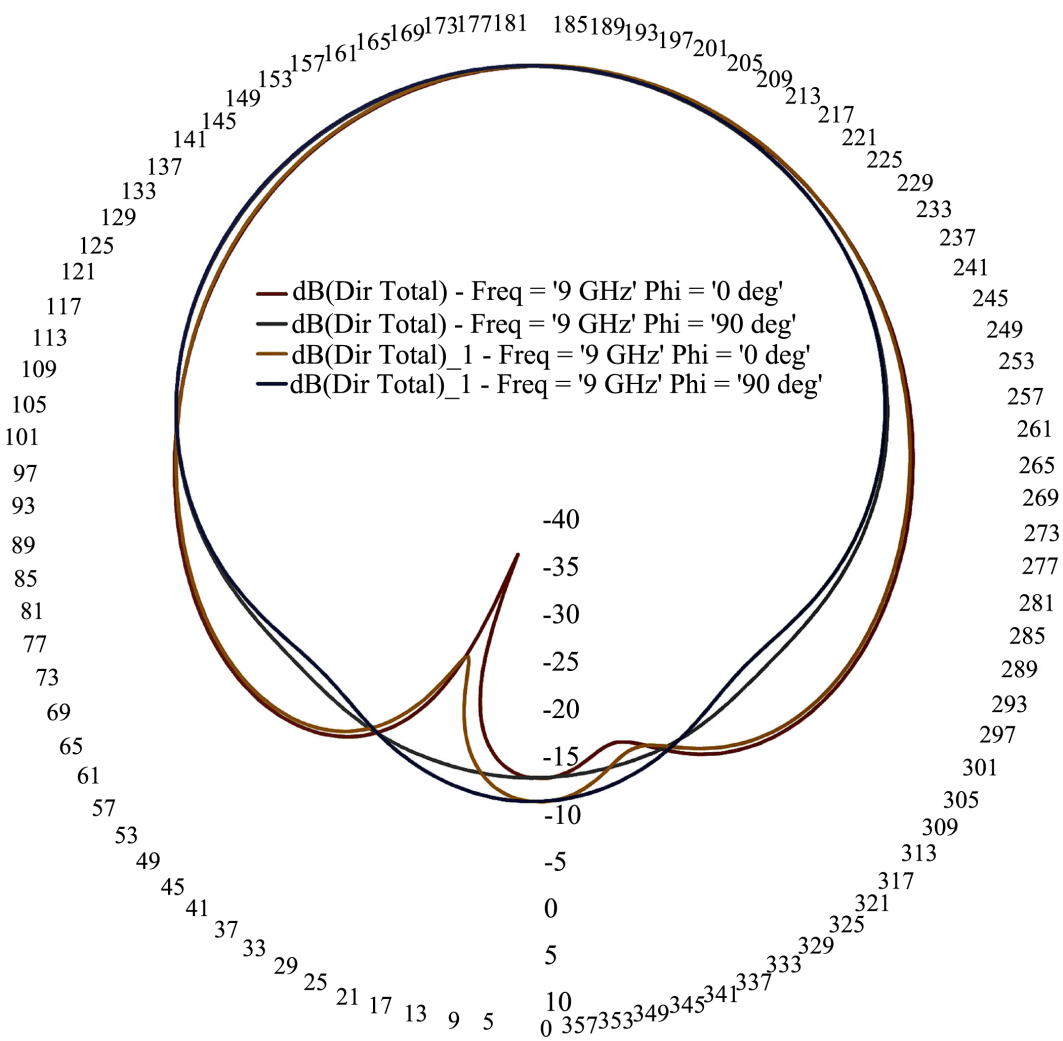

(b)

Figure 6. (a) Gain comparison of with and without MMA loaded MSA; (b) Comparison of directivity with and without MMA loaded MSA. 
The monostatic Radar Cross Section (RCS) is an important design parameter for many applications but accurate RCS prediction of an electrically large structure continues to be a challenging task in Monostatic Radar both Tx and Rx antennas are placed close to each other at the same location Now, Monostatic RCS Analyses with and without MMA loaded MSA and its comparisons are explained. RCS is also an importance aspect of designing this Microstrip antenna with Metamaterial absorber structure as it helps to reduce the radar cross section very effectively for a given resonant frequency.

Here, we have measured Monostatic RCS for loaded arrays of MMA on MSA for $\mathrm{x}$-polarization and y-polarization. It has been found that the values of monostatic RCS have been reduced efficiently. The value of RCS at the exact $9 \mathrm{GHz}$ is found to be $-37.71 \mathrm{~dB}$ for $\mathrm{Phi}=0$ degree and Theta $=0$ degree (x-polarization) and $-38.21 \mathrm{~dB}$ for Phi $=90$ degree and Theta $=0$ degree (y-polarization). The analyses have been given for the loaded MSA with MMA, as it given in Figure 7. Which are efficiently good values but one can reduce the values of RCS by considering a greater number of arrays as mentioned in Table 2 .

Table 2. Dimensions of parameter of unit-cell.

\begin{tabular}{cc}
\hline Parameter & Dimensions $(\mathrm{mm})$ \\
\hline$W$ & 4.25 \\
$L$ & 4.25 \\
$a$ & 0.2 \\
$b$ & 0.4 \\
$p$ & 1.1 \\
$q$ & 2.1 \\
$r$ & 0.7 \\
\hline
\end{tabular}

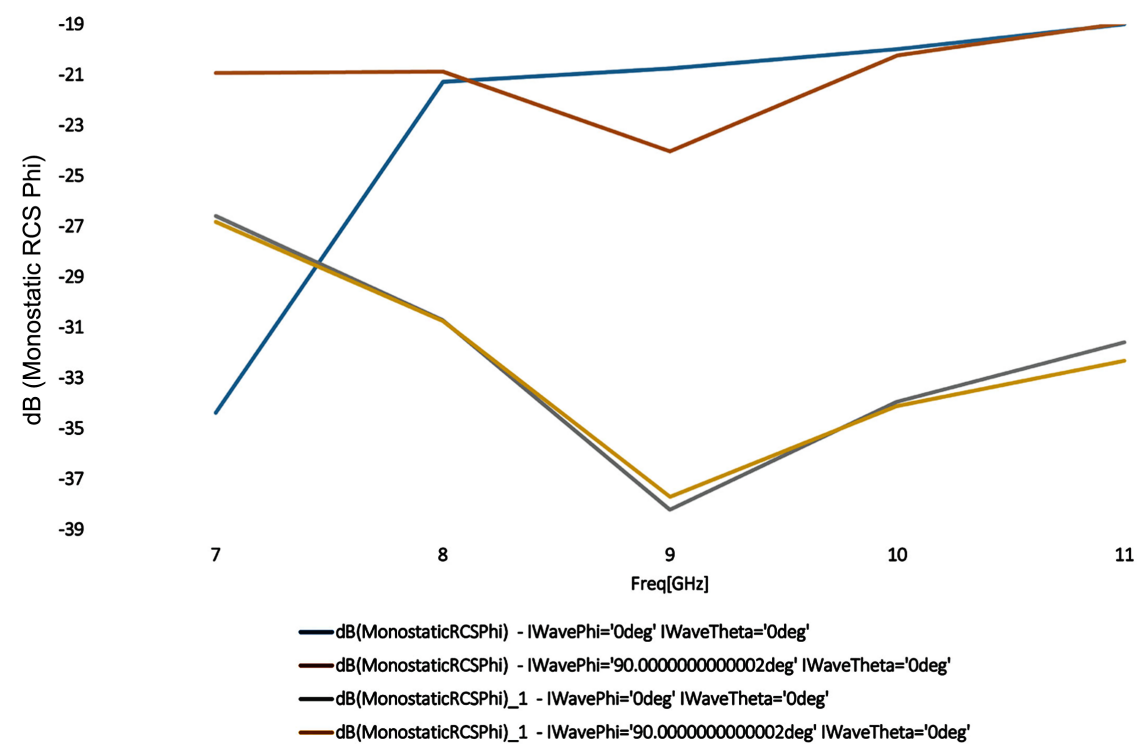

Figure 7. Comparison of with and without MMA loaded MSA monostatic RCS. 


\section{Conclusion}

In this research project work, we have proposed the metamaterial absorber structure operating at $9 \mathrm{GHz}$ of an $\mathrm{x}$-band. An analysis has been given for results of simple microstrip antenna and Metamaterial loaded antenna. Metamaterial unit cell is designed to give absorption at exact $9 \mathrm{GHz}$ and it's nearly $100 \%$. Monostatic RCS had been considered to check the values of RCS and its analytical result of MMA loaded MSA had been given. The values are effectively reducing for RCS. This structure finds its applications in stealth technology for defense applications like, military planes, space aircraft, missiles, ships, and other sensitive vehicles for long distance wireless communication to reduce RCS.

\section{Acknowledgements}

We are thankful to Principal-BVM Engineering College \& TEQIP-III coordinator (World Bank assisted Project) for providing grant for this project. The research was performed and carried out jointly at the Entuple Pvt Ltd and ELARC-Electromagnetics and Antenna Research Centre, which is operated for BVM Engineering College, Vallabh Vidya Nagar-Gujarat-India by Electronics Engineering department.

\section{Conflicts of Interest}

The authors declare no conflicts of interest regarding the publication of this paper.

\section{References}

[1] Walser, R.M. (2001) Electromagnetic Metamaterials. Proceedings. SPIE 4467 Complex Mediums II: Beyond Linear Isotropic Dielectrics, San Diego, Vol. 4467, 1-15. https://doi.org/10.1117/12.432921

[2] Watts, C.M., Liu, X.L. and Padilla, W.J. (2012) Metamaterial Electromagnetic Wave Absorbers. Advanced Materials, 24, OP98-OP120.

[3] Caloz, C. and Itoh, T. (2002) Application of the Transmission Line Theory of Left-Handed (LH) Materials to the Realization of a Microstrip "LH Line". Proceedings of the IEEE Antennas and Propagation Society International Symposium, Vol. 2, 412-415.

[4] Singh, D. and Srivastava, V.M. (2018) Low Radar Cross Section of Patch Antenna Using Shorted Stubs Metamaterial Absorber. International Journal of Microwave and Optical Technology, 13, 194-202.

[5] Balanis, C.A. (2005) Antenna Theory, Analysis and Design. 3rd Edition, John Wiley \& Sons, Hoboken.

[6] Veselago, V.G. (2003) Electrodynamics of Materials with Negative Index of Refraction. Physics-Uspekhi, 173, 790-794.

[7] Pendry, J.B. (2000) Negative Refraction Makes a Perfect Lens. Physical Review Letters, 85, 3966-3969. https://doi.org/10.1103/PhysRevLett.85.3966

[8] Pendry, J.B., Holden, A.J., Robbins, D.J. and Stewart, W.J. (1999) Magnetism from Conductors and Enhanced Nonlinear Phenomena. IEEE Transactions on Microwave Theory and Techniques, 47, 2075-2084. https://doi.org/10.1109/22.798002 
[9] Landy, N.I., Sajuyigbe, S., Mock, J.J., Smith, D.R. and Padilla, W.J. (2008) Perfect Metamaterial Absorber. Physical Review Letters, 100, Article ID: 207402. https://doi.org/10.1103/PhysRevLett.100.207402

[10] Baskey, H.B., Jha, A.K. and Akhtar, M.J. (2014) Design of Metamaterial Based Structure for the Radar Cross Section Reduction of a Microstrip Antenna. 2014 IEEE International Microwave and RF Conference, Bangalore, 15-17 December 2014, 104-107.

[11] Lee, J. and Lim, S. (2011) Bandwidth-Enhanced and Polarisation-Insensitive Metamaterial Absorber Using Double Resonance. Electronics Letters, 47, 8-9. https://doi.org/10.1049/el.2010.2770

[12] Singh, D. and Srivastava, V.M. (2018) Dual Resonances Shorted Stub Circular Rings Metamaterial Absorber. AEU-International Journal of Electronics and Communication, 83, 58-66. https://doi.org/10.1016/j.aeue.2017.08.034

[13] Singh, D. and Srivastava, V.M. (2017) Triple Band Regular Decagon Shaped Metamaterial Absorber for X-Band Applications. IEEE International Conference on Computer Communication and Informatics (ICCCD), Coimbatore, 5-7 January 2017, 1-5. https://doi.org/10.1109/ICCCI.2017.8117766

[14] Li, W.H., Yang, S., Zhang, J.Q., Sai, S., Yuan, H.Y. and Qu, S.B. (2015) The RCS Reduction of Microstrip Antenna Design Based on Multi-Band Metamaterial Absorber. 2015 IEEE MTT-S International Microwave Workshop Series on Advanced Materials and Processes for RF and THz Applications (IMWS-AMP), Suzhou, 1-3 July 2015, 1-3.

[15] Mao, Z., Liu, S., Bian, B., Wang, B., Ma, B., Chen, L. and Xu, J. (2014) Multi-Band Polarization Insensitive Metamaterial Absorber Based on Chinese Ancient Coin-Shaped Structures. Journal of Applied Physics, 115, Article ID: 204505.

[16] Agarwal, M. and Meshram, M.K. (2015) X-Band Metamaterial Absorber with Dual Band/Broadband Absorption Characteristics. 2015 IEEE MTT-S International Microwave and RF Conference (IMaRC), Hyderabad, 10-12 December 2015, 121-124.

[17] Zhai, H., Zhan, C. and Li, Z. (2015) A Triple-Band Ultrathin Metamaterial Absorber with Wide-Angle and Polarization Stability. IEEE Antennas and Wireless Propagation Letters, 14, 241-244. https://doi.org/10.1109/LAWP.2014.2361011

[18] Zhang, Z.-X. and Zhang, J.-C. (2016) RCS Reduction for Patch Antenna Based on Metamaterial Absorber. 2016 Progress in Electromagnetic Research Symposium (PIERS), Shanghai, 8-11 August 2016, 364-368.

[19] Liu, Y., Hao, Y., Jia, Y. and Gong, S.-X. (2014) A Low RCS Dual-Frequency Microstrip Antenna with Complementary Split-Ring Resonators. Progress in Electromagnetics Research, 146, 125-132. https://doi.org/10.2528/PIER14031703

[20] Wang, B.-Y., Liu, S.-B., Bian, B.-R., Mao, Z.-W., Liu, X.-C., Ma, B. and Chen, L. (2014) A Novel Ultrathin and Broadband Microwave Metamaterial Absorber. Journal of Applied Physics, 116, Article ID: 094504. https://doi.org/10.1063/1.4894824

[21] Lu, L., Qu, S.B., Su, X., Shang, Y.B., Zhang, J.Q. and Bai, P. (2013) Simulation and Experiment Demonstration of an Ultra-Thin Wide-Angle Planar Metamaterial Absorber. Physics, 62, Article ID: 208103. https://doi.org/10.7498/aps.62.208103

[22] Jafari, F.S., Naderi, M., Hatami, A. and Zarrabi, F.B. (2019) Microwave Jerusalem Cross Absorber by Metamaterial Split Ring Resonator Load to Obtain Polarization Independence with Triple Band Application. International Journal of Electronics and Communications (AË̈), 101, 138-144.

[23] Jang, H.K., Lee, W.J. and Kim, C.G. (2010) Design and Fabrication of a Microstrip Patch Antenna with a Low RCS in X-Band. Smart Materials and Structures, 20, 1-8. 
https://doi.org/10.1088/0964-1726/20/1/015007

[24] Sharma, A., Gangwar, D., Kanaujia, B.K. and Dwari, S. (2019) Analysis and Design of an Ultra-Thin Metamaterial Absorber and Its Application for In-Band RCS Reduction of Antenna. Journal of Electromagnetic Waves and Applications, 33, 654-667.

[25] Lee, J., Yoon, Y.J. and Lim (2012) Ultra-Thin Polarization Independent Absorber Using Hexagonal Interdigital Metamaterial. Electronics and Telecommunications Research Institute Journal (ETRI), 34, 126-129.

https://doi.org/10.4218/etrij.12.0211.0081 